PROCEEDINGS OF THE

AMERICAN MATHEMATICAL SOCIETY

Volume 126, Number 8, August 1998, Pages 2467-2476

S 0002-9939(98)04142-2

\title{
ON THE DELETED PRODUCT CRITERION FOR EMBEDDABILITY IN $\mathbb{R}^{m}$
}

\author{
A. SKOPENKOV
}

(Communicated by James West)

\begin{abstract}
For a space $K$ let $\tilde{K}=\{(x, y) \in K \times K \mid x \neq y\}$. Let $\mathbb{Z}_{2}$ act on $\tilde{K}$ and on $S^{m-1}$ by exchanging factors and antipodes respectively. We present a new short proof of the following theorem by Weber: For an $n$-polyhedron $K$ and $m \geqslant \frac{3(n+1)}{2}$, if there exists an equivariant map $F: \tilde{K} \rightarrow S^{m-1}$, then $K$ is embeddable in $\mathbb{R}^{m}$. We also prove this theorem for a peanian continuum $K$ and $m=2$. We prove that the theorem is not true for the 3 -adic solenoid $K$ and $m=2$.
\end{abstract}

\section{INTRODUCTION AND FORMULATION OF RESULTS}

This paper is on a classical problem in topology: find necessary and sufficient conditions for either a compactum or a polyhedron $K$ to be embeddable in $\mathbb{R}^{m}$ for a given $m$ (cf. [Wu 65], [RS 96]). All embeddings of polyhedra are assumed to be PL. Let $\tilde{K}=\{(x, y) \in K \times K \mid x \neq y\}$ be the deleted product of $K$. Let $\mathbb{Z}_{2}$ act on $\tilde{K}$ and on $S^{m-1}$ by exchanging factors and antipodes respectively. If $f: K \rightarrow \mathbb{R}^{m}$ is an embedding, then there exists an equivariant map $\tilde{f}: \tilde{K} \rightarrow S^{m-1}$, defined by $\tilde{f}(x, y)=\frac{f(x)-f(y)}{\|f(x)-f(y)\|}$. The existence of an equivariant map $F: \tilde{K} \rightarrow S^{m-1}$ implies embeddability of $K$ in $\mathbb{R}^{m}$ for a Diff $n$-manifold or $n$-polyhedron $K$ and $m \geq \frac{3(n+1)}{2}$ [Hae 63], [We 67] (see also [MS 67], [Har 69], [Hu 88], [SS 92], [Sk 97], [SSS 97]).

For a triangulation $T$ of $K$ denote $\tilde{T}=\{\sigma \times \tau \in T \times T \mid \sigma \cap \tau=\emptyset\}$. A map $f: K \rightarrow \mathbb{R}^{m}$ is called an almost-embedding (w.r.t. $T$ ) if $f \sigma \cap f \tau=\emptyset$ for each $\sigma \times \tau \in \tilde{T}$ [FKT 94]. The proof in [We 67] consists of two parts: generalized Whitney construction (from the existence of $F$ follows the existence of an almost-embedding $K \rightarrow \mathbb{R}^{m}$ ) and generalized van Kampen construction (from the existence of an almost-embedding follows the existence of an embedding $K \rightarrow \mathbb{R}^{m}$ ). The second (hardest) part contains a mistake [We 67, p.24, lines 9 and 18] which is seemingly just a technical one and can be eliminated using the same ideas. We present a new and shorter proof of this part without relying on Freudenthal's Suspension Theorem. It is also a direct proof of a corollary of Weber's theorem.

Received by the editors April 12, 1995 and, in revised form, January 3, 1997.

1991 Mathematics Subject Classification. Primary 57Q35, 54C25; Secondary 55S15, 57Q30, 57Q65, 57Q40.

Key words and phrases. Embedding, deleted product, engulfing, quasi-embedding, metastable case, peanian continua, 3-adic solenoid, relative regular neighborhood.

Supported by the Russian Fundamental Research Foundation, Grant No 96-01-01166A.

(C) 1998 American Mathematical Society 
Theorem 1.1 ([We 67]). If $K$ is an n-polyhedron with a triangulation $T, m \geq$ $\frac{3(n+1)}{2}$ and $\varphi: K \rightarrow \mathbb{R}^{m}$ is a map such that $\varphi \sigma \cap \varphi \tau=\emptyset$ for each $\sigma \times \tau \in \tilde{T}$, then there is an embedding $f: K \rightarrow \mathbb{R}^{m}$ such that $\left.\tilde{f}\right|_{\tilde{T}}$ is equivariantly homotopic to $\left.\tilde{\varphi}\right|_{\tilde{T}}$.

Corollary 1.2 ([We 74]). For an n-polyhedron $K$ and $m \geqslant \frac{3(n+1)}{2}$, if $K$ is quasiembeddable in $\mathbb{R}^{m}$ then $K$ is embeddable in $\mathbb{R}^{m}$.

A polyhedron $K$ is called quasi-embeddable in $\mathbb{R}^{m}$ if for each triangulation $T$ of it there exists an almost-embedding of $K$ in $\mathbb{R}^{m}$ (w.r.t. $T$ ). This definition is non-standard, but equivalent to the standard one.

We prove Theorem 1.1 first under the additional assumption that $\left.\varphi\right|_{\alpha}$ is an embedding for each $\alpha \in T$. Note that in the second part of Weber's proof we already have this assumption. At the end of section 2 we show how to drop it. We use induction on simplices. The induction step is modification of $\left.\varphi\right|_{\sigma^{p} \cup \sigma^{q}}$ to an embedding for some $\sigma^{p}, \sigma^{q} \in T$ such that $\sigma^{p} \neq \sigma^{q}$ and $\sigma^{p} \cap \sigma^{q} \neq \emptyset$. Using relative regular neighborhoods [Co 69], we engulf the intersection $\varphi \sigma^{p} \cap \varphi \sigma^{q}$ into a PL-ball $D^{m}$ so that

$$
\left(D^{m} \cap \varphi\left(\sigma^{p} \cup \sigma^{q}\right), \partial D^{m} \cap \varphi\left(\sigma^{p} \cup \sigma^{q}\right)\right) \cong\left(D^{p} \bigcup_{D^{r}} D^{q}, \partial D^{p} \bigcup_{D^{r}} \partial D^{q}\right),
$$

where $D^{r}$ is unknotted in $\partial D^{p}$ and in $\partial D^{q}$. Then by $[\mathrm{Li} 65$, th.9 and the discussion before its statement] and since $m-3 \geqslant p, q$, it follows that $\partial D^{p} \bigcup_{D^{r}} \partial D^{q}$ is unknotted in $\partial D^{m}$. Therefore we can alter $\varphi$ on $\varphi^{-1} D^{m}$ to an embedding on $\sigma^{p} \cup \sigma^{q}$. So as not to destroy improvements from previous steps, we need general position and hence $m \geqslant \frac{3(n+1)}{2}$. Our proof does not use Freudenthal's Suspension theorem; however the dimension restriction $m \geqslant \frac{3(n+1)}{2}$ is still necessary. For $m=2 n$ this is [FKT 94, 1.5]. For a controlled version of Weber's theorem and of the above proof, see [RS 97].

A finite 2-polyhedon is embeddable in $\mathbb{R}^{2}$ if and only if it does not contain any of the three subpolyhedra $K_{5}, K_{33}, U$ (Figure 1) [Ku 30], [HJ 64], [MS 66] (see also [Th 81], [Sa 91], [Ma 97]). A peanian continuum is embeddable in $\mathbb{R}^{2}$ if and only if it does not contain any of the four subcontinua $K_{5}, K_{33}, P^{\prime}, Q^{\prime}$ (Figure 1 ; for the description of $P^{\prime}$ and $Q^{\prime}$ see $\S 3.1$ ) [Cl 34], [Cl 37]. We prove a corollary of this theorem:

Theorem 1.3 (for graphs and 2-polyhedra see [Wu 65], [SSS 97]). For a peanian continuum $K$, if there exists an equivariant map $F: \tilde{K} \rightarrow S^{1}$, then $K$ is embeddable in $\mathbb{R}^{2}$.

Example 1.4. For the 3-adic solenoid $\Sigma$, there exists an equivariant map $F: \tilde{\Sigma} \rightarrow$ $S^{1}$, but $\Sigma$ is not embeddable in $\mathbb{R}^{2}$.

Recall that the 3-adic solenoid is the intersection of an infinite sequence of filled tori, each of them inscribed into the previous one with degree 3 . The construction of Example 1.4 is based on the inverse limits technique (cf. [RS 97, example 1.5]).

Conjecture 1.5. There exists a non-planar tree-like continuum $K$ for which there is an equivariant map $\tilde{K} \rightarrow S^{1}$.

\section{Proof of TheOREM 1.1}

We use the notation of [RS 72]. The upper index of a polyhedron shows its dimension. Order simplices of $T$ with respect to increasing dimension. We use 

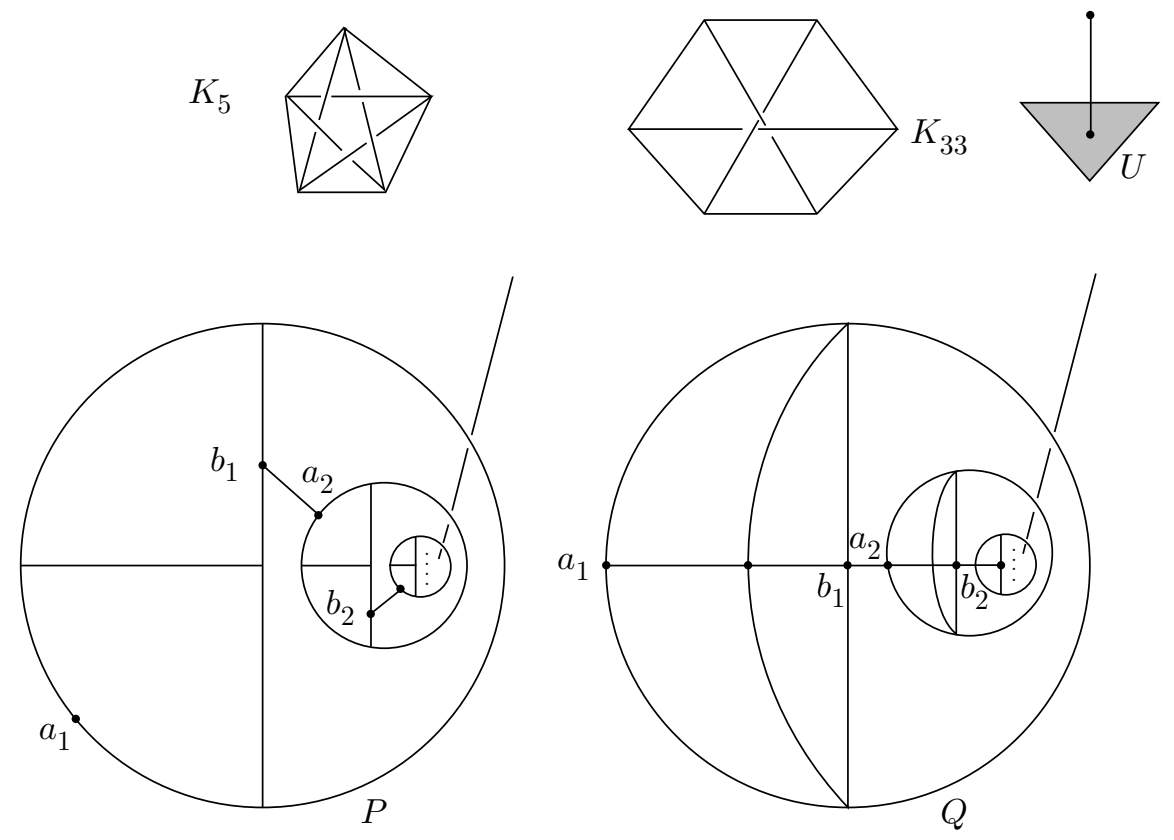

FiguRE 1

the lexicographic order on $T \times T$. Suppose first that $\left.\varphi\right|_{\alpha}$ is an embedding for each $\alpha \in T$. Theorem 1.1 follows from Proposition 2.1 below for $\sigma^{p}=\sigma^{q}=$ (the last simplex of $T$ ).

Proposition 2.1. For each $\sigma^{p} \times \sigma^{q} \in T \times T$ such that $\sigma^{p} \geqslant \sigma^{q}$ there exists a $P L$ map $f: K \rightarrow \mathbb{R}^{m}$ such that

(2.1.1) $f \alpha \cap f \beta=\emptyset$ for each $\alpha \times \beta \in \tilde{T}$;

(2.1.2) $\left.f\right|_{\alpha}$ is an embedding for each $\alpha \in T$;

(2.1.3) $\left.\tilde{f}\right|_{\tilde{T}}$ is equivariantly homotopic to $\left.\tilde{\varphi}\right|_{\tilde{T}}$;

(2.1.4) $f \alpha \cap f \beta=f(\alpha \cap \beta)$ for $(\alpha, \beta)<\left(\sigma^{p}, \sigma^{q}\right)$.

Proof. The map $\varphi$ already satisfies (2.1.1)-(2.1.3). We achieve (2.1.4) by induction on $\left(\sigma^{p}, \sigma^{q}\right)$. Base $\sigma^{p}=$ (the first simplex of $T$ ) follows by taking $f=\varphi$. Now assume that $f$ satisfies (2.1.1)-(2.1.4). We may assume that $f$ is in general position. Suppose that $p+q \geqslant m$ and $\sigma^{q} \not \subset \sigma^{p}$ and $\sigma^{q} \cup \sigma^{p}$ is not contained in the boundary of some simplex of $T$ (otherwise the inductive step holds either by general position or by the induction hypothesis). Let $D^{r}=f\left(\sigma^{p} \cap \sigma^{q}\right)$. By (2.1.2), $D^{r}$ is a PL-ball.

Ball Lemma 2.2. There are PL-balls $D^{p}, D^{q}, D^{m} \subset \mathbb{R}^{m}$ such that

(2.2.1) $D^{p} \subset D^{r} \cup f \dot{\circ}^{p}$ and $D^{q} \subset D^{r} \cup f \dot{\circ}^{q}$;

(2.2.2) $D^{p}=D^{m} \cap f \sigma^{p}$ and $D^{q}=D^{m} \cap f \sigma^{q}$ are properly embedded in $D^{m}$;

(2.2.3) $D^{r}=\partial D^{p} \cap \partial D^{q}$;

(2.2.4) $D^{r}$ is unknotted in $\partial D^{p}$ and in $\partial D^{q}$;

(2.2.5) $\Sigma=\mathrm{Cl}\left(\left(f \sigma^{p} \cap f \sigma^{q}\right)-D^{r}\right) \subset \stackrel{\circ}{D}^{m} \cup D^{r}$;

(2.2.6) $D^{m} \cap X \subset D^{r}$, where $X=\bigcup f\left\{\alpha \in T \mid \alpha \cap \sigma^{p}=\emptyset\right.$ or $\left.\alpha<\sigma^{q}\right\}$. 
Proof of Proposition 2.1 modulo Ball Lemma 2.2. Take PL-balls $D^{p}, D^{q}, D^{m}$ given by the Ball Lemma. Recall [Li 65 , th. 9 and the discussion before its statement]: If $m-3 \geqslant p, q, S^{p}, S^{q} \subset S^{m}$ and $S^{p} \cap S^{q}=D^{r}$, where $D^{r}$ is unknotted in $S^{p}$ and in $S^{q}$, then $S^{p} \cup S^{q}$ is unknotted in $S^{m}$. Hence we may assume that $\partial D^{p} \cup_{D^{r}} \partial D^{q} \subset$ $\partial D^{m}$ standardly. By the relative Unknotting Balls Theorem [Ze 66, Corollary 1 to Theorem 9] we may assume that $\left(D^{q}, \partial D^{q}\right) \subset\left(D^{m}, \partial D^{m}\right)$ standardly. Hence the embedding $\partial D^{p} \subset \partial D^{m}$ can be extended to a new embedding of $D^{p}$ into $\left(\stackrel{\circ}{D}^{m} \backslash D^{q}\right) \cup \partial D^{p}$. By the relative Unknotting Balls Theorem this new embedding is ambiently isotopic to $D^{p} \subset D^{m}$ rel $\partial D^{m}$. So there is an isotopy $h_{t}: D^{m} \rightarrow$ $D^{m}$ rel $\partial D^{m}$ such that $D^{q} \cap h_{1} D^{p}=D^{r}$. Define a map $f^{+}: K \rightarrow \mathbb{R}^{m}$ as

$$
f^{+}(x)= \begin{cases}h_{1}(f(x)), & \text { if } f(x) \in D^{m} \text { and } x \in \gamma \text { for some } \gamma \text { containing } \sigma^{p}, \\ f(x), & \text { otherwise. }\end{cases}
$$

Evidently, $f^{+}$satisfies (2.1.1)-(2.1.3). Since $\sigma^{p} \cup \sigma^{q}$ is not contained in the boundary of some simplex of $T, D^{q} \cap h_{1} D^{p}=D^{r}$, and by (2.2.5) and (2.2.6), it follows that $f^{+}$satisfies also $(2.1 .4)$ for $(\alpha, \beta) \leqslant\left(\sigma^{p}, \sigma^{q}\right)$. The induction step is proved.

\subsection{Proof of the Ball Lemma.}

Preliminary constructions (cf. [We 67, §6a]; see Figure 2). Let us make two conventions on the triangulations. First, for polyhedra $M \supset Z \supset Y$ the notation $R_{M}(Z, Y)$ means 'a regular neighborhood of $Z \operatorname{rel} Y$ in $M$ in some small triangulation of $\mathbb{R}^{m}$, when it first appears, and 'the regular neighborhood of $Z \operatorname{rel} Y$ in $M^{\prime}$, after the first appearance. Second, regular neighborhoods defining $D^{p}, D^{q}$ and $D^{m}$ below should be in restrictions of the same triangulation of $\mathbb{R}^{m}$. Also, $R_{M}(Z)=R_{M}(Z, \emptyset)$. Let $S$ be the link of some $r$-simplex from $\stackrel{\circ}{D}^{r}$ in some small triangulation of $\mathbb{R}^{m}$. Then $S$ is a PL $(m-r-1)$-sphere and $R_{\mathbb{R}^{m}}\left(D^{r}, \partial D^{r}\right) \cong S * D^{r}$. By (2.1.2) $R_{\mathbb{R}^{m}}\left(D^{r}, \partial D^{r}\right) \cap f \alpha=R_{f \alpha}\left(D^{r}, \partial D^{r}\right)$ goes to $(S \cap f \alpha) * D^{r}$ under this homeomorphism for each $\alpha \in T$ (for $\alpha \not \supset \sigma^{p} \cap \sigma^{q}$ each of these three sets is empty). Also $S \cap f \alpha$ is a PL $(\operatorname{dim} \alpha-r-1)$-ball for each $\alpha \in T, \alpha \supset \sigma^{p} \cap \sigma^{q}$.

Take distinct points $a \in\left(S \cap f \stackrel{\circ}{\sigma}^{p}\right) \backslash X$ and $b \in\left(S \cap f \stackrel{\circ}{\sigma}^{q}\right) \backslash X$. Since $m-r-1 \geqslant 2$ and $(n-r-1)+1<m-r-1$, then by general position there exists an $\operatorname{arc} l \subset S$, joining $a$ and $b$, such that $l \cap X=\emptyset, l \cap f \sigma^{p}=a$ and $l \cap f \sigma^{q}=b$. Let $\beta=R_{S}(l) * D^{r}$. Then $\beta \cap f \sigma^{p}$ and $\beta \cap f \sigma^{q}$ are PL $p$ - and $q$-balls.

Collapsing Lemma 2.4 (follows from [Co 69, th. 3.1 and add. 3.4]). If $A$ and $F$ are regular neigborhoods of a polyhedron $Z$ in a $P L$-manifold $M \operatorname{rel} Y$ and $A \subset F$, then $F \searrow A \operatorname{rel} Y$.

Construction of $D^{p}$ and $D^{q}$ (Figure 3). By the induction hypothesis, $f \sigma^{p} \cap f \partial \sigma^{q}=$ $f \partial \sigma^{p} \cap f \sigma^{q}=D^{r}$. Hence $\Sigma \subset\left(f \dot{\sigma}^{p} \cap f \dot{\circ}^{q}\right) \cup D^{r}$. Both $f \sigma^{p}$ and $\left(S * D^{r}\right) \cap$ $f \sigma^{p}=\left(S \cap f \sigma^{p}\right) * D^{r}$ are regular neighborhoods of $D^{r}$ rel $\partial D^{r}$ in $f \sigma^{p}$. Then by Collapsing Lemma 2.4, $f \sigma^{p} \searrow\left(S \cap f \sigma^{p}\right) * D^{r}$ rel $D^{r}$. Both $S \cap f \sigma^{p}$ and $R_{S \cap f \sigma^{p}}(a)$ are regular neighborhoods of $a$ in $S \cap f \sigma^{p}$. Then by Collapsing Lemma 2.4, $S \cap f \sigma^{p} \searrow$ $R_{S \cap f \sigma^{p}}(a)$. Hence

$$
\left(S \cap f \sigma^{p}\right) * D^{r} \searrow R_{S \cap f \sigma^{p}}(a) * D^{r}=\beta \cap f \sigma^{p} \operatorname{rel} D^{r} .
$$

Let $C_{1}$ be the trail of $\Sigma$ under the above sequence of collapses

$$
f \sigma^{p} \searrow\left(S \cap f \sigma^{p}\right) * D^{r} \searrow \beta \cap f \sigma^{p} \operatorname{rel} D^{r}
$$




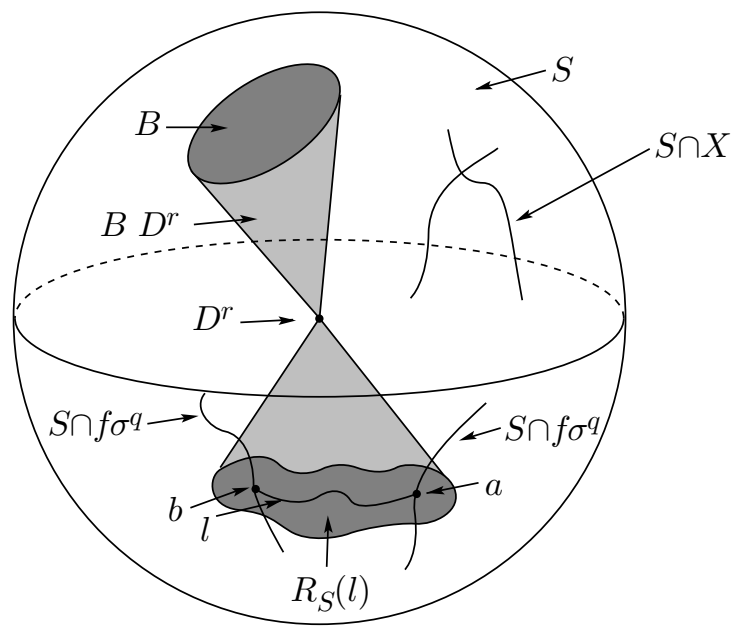

FiguRE 2

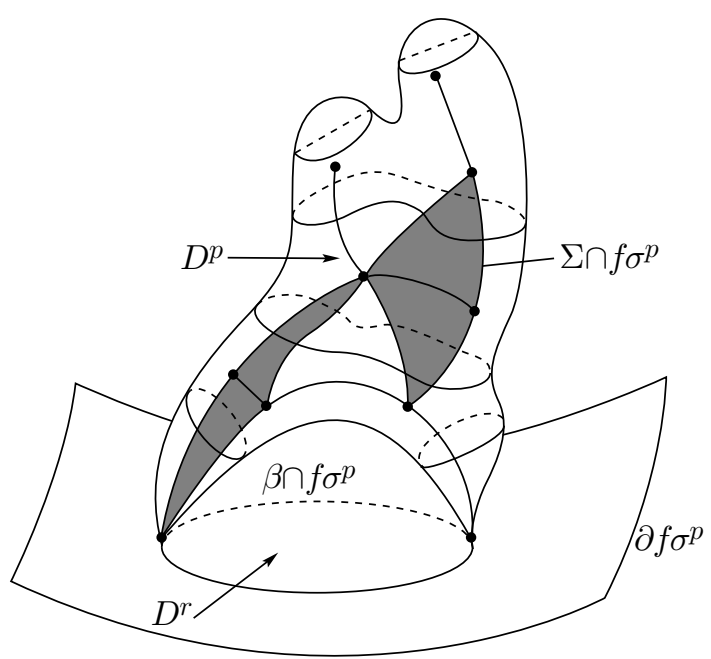

FiguRE 3

that is in general position. Let $D^{p}=R_{f \sigma^{p}}\left(\left(\beta \cap f \sigma^{p}\right) \cup C_{1}, D^{r}\right)$. Then (2.2.1) and (2.2.4) are true for $D^{p}$, and

(2.3.1) $C_{1} \subset f \sigma^{p}$

(2.3.2) $\Sigma \subset\left(\beta \cap f \sigma^{p}\right) \cup C_{1}$;

(2.3.3) $D^{p}$ is a PL $p$-ball;

(2.3.4) $C_{1} \cap X=\emptyset$;

(2.3.5) $D^{p} \cap X \subset D^{r}$;

(2.3.6) $C_{1} \cap f \sigma^{q}=\Sigma$.

Actually, (2.3.1) and (2.3.2) are obvious. Since $\Sigma \subset D^{r} \cup f^{\circ}{ }^{p}$, it follows that $C_{1} \subset D^{r} \cup f \stackrel{\circ}{\sigma}^{p}$; hence $(2.2 .1)$ is true. Since $f \sigma^{p}$ is a PL-manifold and $f \sigma^{p} \searrow$ $\left(\beta \cap f \sigma^{p}\right) \cup C_{1} \operatorname{rel} D^{r}$, then $f \sigma^{p}$ is a regular neighborhood of $\left(\beta \cap f \sigma^{p}\right) \cup C_{1}$ in $f \sigma^{p} \operatorname{rel} D^{r}\left[\right.$ Co 69 , th. 9.1]. Then by [Co 69, th. 3.1] there is an isotopy $G_{t}$ : 
$f \sigma^{p} \rightarrow f \sigma^{p} \operatorname{rel}\left(\beta \cap f \sigma^{p}\right) \cup C_{1}$ between $G_{0}=$ id and a homeomorphism $G_{1}$ of $f \sigma^{p}$ onto $D^{r} \operatorname{rel}\left(\beta \cap f \sigma^{p}\right) \cup C_{1}$. This implies (2.3.3). Moreover, $\left.G_{1}\right|_{\partial f \sigma^{p}}$ is a homeomorphism of $\partial f \sigma^{p}$ onto $\partial D^{p}$ rel $D^{r}$. Since $D^{r}$ is unknotted in $\partial f \sigma^{p}$, then (2.2.4) is true for $D^{p}$. By general position, $\operatorname{dim} \Sigma \leqslant 2 n-m$. Then $\operatorname{dim} C_{1} \leqslant 2 n-m+1$. By general position and since $n+(2 n-m)<m, \Sigma \cap X=\emptyset$. Again general position and $n+(2 n-m+1)<m$ imply (2.3.4). Since $l \cap X=\emptyset$, it follows that $\beta \cap f \sigma^{p} \cap X=D^{r}$. This and (2.3.4) imply (2.3.5). By definition of relative collapse, $C_{1} \cap D^{r}=\Sigma \cap D^{r}$. Therefore, by general position $(n+(2 n-m+2)<m)$, we have $(2.3 .6)$.

Analogously we can construct polyhedra $C_{2}$ and $D^{q}$ such that (2.2.1), (2.2.4) and (2.3.1)-(2.3.6) are true for $C_{1} \rightarrow C_{2}$ and $p \rightarrow q$.

Construction of $D^{m}$ (Figure 2). Take a PL $(m-r-1)$-ball $B \subset S-\left(l \cup f \sigma^{p} \cup f \sigma^{q}\right)$. By [Al 30], $S-\stackrel{\circ}{B}$ is a PL $(m-r-1)$-ball and $\sigma^{m}=\left(\mathbb{R}^{m} \cup \infty\right)-\operatorname{Int}\left(B * D^{r}\right)$ is a PL $m$-ball. By (2.3.1), $C_{1} \cap\left(S * D^{r}\right) \subset\left(S \cap f \sigma^{p}\right) * D^{r}$. Then $C_{1} \cap \operatorname{Int}\left(B * D^{r}\right)=\emptyset$ and hence $C_{1} \subset \stackrel{\circ}{\sigma}^{m} \cup D^{r}$. Analogously, $C_{2} \subset \stackrel{\circ}{\sigma}^{m} \cup D^{r}$. Then, similarly to the construction of $D^{p}$ and $D^{q}$, let $C$ be a trail of $C_{1} \cap C_{2}$ under a sequence of collapses

$$
\sigma^{m} \searrow \sigma^{m} \cap\left(S * D^{r}\right)=(S-\stackrel{\circ}{B}) * D^{r} \searrow R_{S}(l) * D^{r}=\beta \operatorname{rel} D^{r}
$$

that is in general position. Analogously to (2.3.1)-(2.3.3) it is proved that $C \subset$ $\sigma^{m} \cup D^{r}, C_{1} \cup C_{2} \subset \beta \cup C$ and $D^{m}=R_{\sigma^{m}}\left(\beta \cup C, D^{r}\right)$ is a PL $m$-ball. Analogously to (2.3.4), using (2.3.4) and $n+(2 n-m+2)<m$, we can prove that $C \cap X=\emptyset$. Then (2.2.6) is proved analogously to (2.3.5). (2.3.6) and general position imply

$$
C \cap f \sigma^{q}=\left(C_{1} \cup C_{2}\right) \cap f \sigma^{q}=C_{2} \cup\left(C_{1} \cap f \sigma^{q}\right)=C_{2} \cup \Sigma=C_{2} .
$$

Analogously $C \cap f \sigma^{p}=C_{1}$. Therefore $(\beta \cup C) \cap f \sigma^{p}=\left(\beta \cap f \sigma^{p}\right) \cup C_{1}$ and $(\beta \cup C) \cap f \sigma^{q}=\left(\beta \cap f \sigma^{q}\right) \cup C_{2}$. Therefore, since $D^{p}, D^{q}$ and $D^{m}$ are regular neighborhoods rel $D^{r}$ of $\left(\beta \cap f \sigma^{p}\right) \cup C_{1},\left(\beta \cap f \sigma^{q}\right) \cup C_{2}$ and $\beta \cup C$ in restrictions of the same triangulation of $\mathbb{R}^{m}$ to $f \sigma^{p}, f \sigma^{q}$ and $\sigma^{m}$, we get (2.2.2). By (2.3.2) and the definitions of $D^{p}, D^{q}, \Sigma$,

$$
\left(\partial D^{p}-D^{r}\right) \cap\left(\partial D^{q}-D^{r}\right) \subset\left(f \dot{\circ}^{p}-\Sigma\right) \cap\left(f \dot{\sigma}^{q}-\Sigma\right)=\emptyset .
$$

Hence (2.2.3) is true. By (2.3.1) we have $\Sigma \subset\left(\beta \cap f \sigma^{p}\right) \cup C_{1} \subset \beta \cup C \subset \stackrel{\circ}{ }^{m} \cup D^{r}$, so $(2.2 .5)$ is true.

2.5. Dropping the additional assumption. It sufficies to make the following modifications in section 2. Condition (2.1.2) is altered to ' $\left.f\right|_{\alpha}$ is an embedding for each $\alpha \leq \sigma^{p}$. Actually, in the proof of Proposition 2.1 we used this weaker property rather than (2.1.2). In the inductive step of Proposition 2.1 we may assume that $\sigma^{q}$ is not a proper subset of $\sigma^{p}$ (otherwise (2.1.4) for $(\alpha, \beta)=\left(\sigma^{p}, \sigma^{q}\right)$ follows from (2.1.2)). The proof splits into two cases.

In the case $\sigma^{q} \not \subset \sigma^{p}$ the proof is as above. Only the following modifications are necessary. In the proof modulo the Ball Lemma, before construction of $f^{+}$, take a function $\xi: K \rightarrow[0,1]$ such that $\xi\left(D^{p}\right)=1, \xi(x) \neq 0$ only for $x \in$ $R_{K}\left(D^{p}, D^{r}\right) \cap f^{-1} D^{m}$ and $\xi$ continuous on $K-D^{r}$. The map $f^{+}: K \rightarrow \mathbb{R}^{m}$ is defined as $f^{+}(x)=f(x)+\left(h_{1}(f(x))-f(x)\right) \xi(x)$. Since $D^{r} \subset \partial D^{m}$, then $f x=h_{1} x$ on $f^{-1} D^{r}$; hence $f^{+}$is continuous.

In the case $\sigma^{q}=$ (the first simplex of $T$ ), we need to achieve condition (2.1.2) for $\alpha=\sigma^{p}$. We have $\left(\alpha, \sigma^{p}\right) \geq\left(\sigma^{q}, \sigma^{p}\right)$ if $\alpha \cap \sigma^{p} \neq \emptyset$. Take a PL ball $D^{m}$ given by the Ball Lemma 2.6 below. By the Unknotting Balls Theorem [Ze 63], the map $\left.f\right|_{\sigma^{p}}: \sigma^{p} \rightarrow D^{m}$ is homotopic rel $\partial \sigma^{p}$ to an embedding $h: \sigma^{p} \rightarrow D^{m}$. Let 


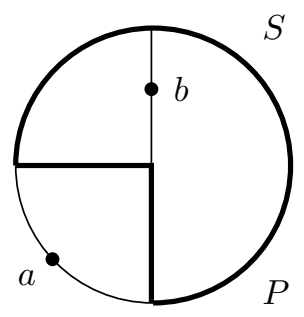

a)

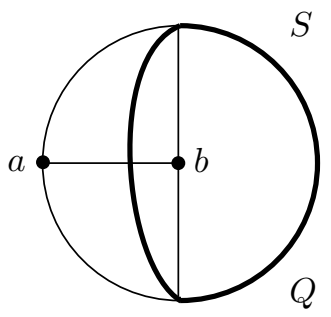

b)

FIGURE 4

$N=R_{K}\left(\sigma^{p}, \partial \sigma^{p}\right)$, so that $f N \cap X=\emptyset$. Let $f^{+}: K \rightarrow \mathbb{R}^{m}$ be any map equal to $f$ on $\mathrm{Cl}(K-N)$, to $h$ on $\sigma^{p}$ and such that $f^{+} N \cap X=\emptyset$. Evidently, $f^{+}$satisfies (2.1.1)-(2.1.4). Since $\sigma^{q}=$ (the first simplex of $T$ ), by general position it follows that (2.1.4) holds for $(\alpha, \beta)=\left(\sigma^{q}, \sigma^{p}\right)$. The induction step is proved.

Ball Lemma 2.6. There is a $P L$ ball $D^{m} \subset \mathbb{R}^{m}$ such that $f \sigma^{p} \subset \stackrel{\circ}{D^{m}}$ and $D^{m} \cap$ $X=\emptyset$, where $X=f \cup\left\{\alpha \in T \mid \alpha \cap \sigma^{p}=\emptyset\right\}$.

Proof. Let $C_{1} \subset \sigma^{p}$ be a collapsible polyhedron of dimension at most $2 p-m+1$ containing $S\left(\left.f\right|_{\sigma^{p}}\right)$. Let $C \subset \mathbb{R}^{m}$ be a collapsible polyhedron of dimension at most $2 p-m+2$ containing $f C_{1}$. Since $(2 p-m+1)+n<m$, by general position it follows that $f^{-1} C=C_{1}$. From (2.1.1) it follows that $f \sigma^{p} \cap X=\emptyset$, hence similarly by general position, $C \cap X=\emptyset$. Since $\left.f\right|_{\sigma^{p}-C_{1}}$ is an embedding and $\sigma^{p} \searrow C_{1}$, it follows that $C \cup f \sigma^{p} \searrow C \searrow *$. Then $D^{m}=R_{\mathbb{R}^{m}}\left(C \cup f \sigma^{p}\right)$ is the required ball.

\section{Planar case}

3.1. Construction of Claytor's continua. Let $P$ and $Q$ be the graphs shown on Figure 4a, b. Let $a, b$ and $S$ be two points and a simple closed curve in $P$, shown on Figure 4a. Let $\left\{P_{n}\right\}$ be a null-sequence of copies of $P$, converging to a point $0 \notin \coprod_{n=1}^{\infty} P_{n}$. Denote elements of $P_{n}$, corresponding to $a, b$ and $S$, by $a_{n}, b_{n}$ and $S_{n}$. Let $\left\{I_{n}\right\}$ be a null-sequence of arcs, joining $b_{n} \in P_{n}$ to $a_{n+1} \in P_{n+1}$ and converging to the same point $0 \notin \coprod_{n=1}^{\infty} I_{n}$. Then $P^{\prime}=[0,1] \cup \bigcup_{n=1}^{\infty}\left(P_{n} \cup I_{n}\right)$. $Q^{\prime}$ is defined similarly, replacing $P$ by $Q$ and Figure 4 a by Figure $4 \mathrm{~b}$.

3.2. Proof of Theorem 1.3. It suffices to prove that there are no equivariant maps $\tilde{P}^{\prime} \rightarrow S^{1}$ and $\tilde{Q}^{\prime} \rightarrow S^{1}$. To prove it for $\tilde{P}^{\prime}$ (for $\tilde{Q}^{\prime}$ the proof is analogous), suppose to the contrary that $F: \tilde{P}^{\prime} \rightarrow S^{1}$ is an equivariant map. Since $S_{n}$ converges to 0 , then for sufficiently great $n,\left.F\right|_{S_{n} \times 1}$ is 'close' to $\left.F\right|_{0 \times 1}$ and hence inessential. Taking a subsequence of $\left\{P_{n}\right\}$, we may assume that $\left.F\right|_{S_{n} \times 1}$ is inessential for each $n$. Since $\left.F\right|_{S_{n} \times t}$ is a 'homotopy' between $\left.F\right|_{S_{n} \times 0}$ and $\left.F\right|_{S_{n} \times 1}$, then $\left.F\right|_{S_{n} \times 0}$ is inessential, too. Since $S_{n}$ converges to 0 , then for each $n$ and sufficiently great $m(n),\left.F\right|_{S_{n} \times S_{m(n)}}$ is inessential. Taking a subsequence of $\left\{P_{n}\right\}$, we may assume that $\left.F\right|_{S_{n} \times S_{m}}$ is inessential for each $n, m$. 
Let $J_{n}$ be an arc joining $a_{n}$ to $b_{n}$ and such that $J_{n} \cap P_{n}=\left\{a_{n}, b_{n}\right\}$. Then $P_{n} \cup$ $J_{n} \cong K_{33}$; hence there is no equivariant map $\widetilde{P_{n} \cup J_{n}} \rightarrow S^{1}$ [Wu 65]. Therefore $\left.F\right|_{\tilde{P}_{n}}$ is not equivariantly extendable over $\tilde{P}_{n} \cup S_{n} \times J_{n} \cup J_{n} \times S_{n}$, and hence $\left.F\right|_{S_{n} \times a_{n}}$ and $\left.F\right|_{S_{n} \times b_{n}}$ are not homotopic. In particular, $\left.F\right|_{S_{n} \times a_{n}}$ and $\left.F\right|_{S_{n} \times b_{n}}$ cannot be both inessential. But $\left.F\right|_{S_{2} \times S_{1}}$ and $\left.F\right|_{S_{2} \times S_{3}}$ are both inessential. Since $\left.\left.\left.F\right|_{S_{2} \times a_{2}} \simeq F\right|_{S_{2} \times b_{1}} \simeq F\right|_{S_{2} \times \text { a point in } S_{1}}$, then $\left.F\right|_{S_{2} \times a_{2}}$ is inessential. Analogously, $\left.F\right|_{S_{2} \times b_{2}}$ is inessential, which is a contradiction.

3.3. Construction of an equivariant map $\tilde{\Sigma} \rightarrow S^{1}$. Let $S^{1}=\{z \in \mathbb{C}|| z \mid=$ $1\}$. Define $p: S^{1} \rightarrow S^{1}$ by $p(z)=z^{3}$. We have

$$
\Sigma=\left\{\left(x_{1}, x_{2}, \ldots\right) \mid x_{i} \in S^{1}, p x_{i+1}=x_{i}\right\}
$$

and

$$
\begin{array}{r}
\tilde{\Sigma}=\left\{\left(x_{1}, y_{1}, x_{2}, y_{2}, \ldots\right) \mid x_{i}, y_{i} \in S^{1}, p x_{i+1}=x_{i}, p y_{i+1}=y_{i} \text { for each } i\right. \\
\text { and } \left.x_{n} \neq y_{n} \text { for some } n\right\}
\end{array}
$$

with the Tikhonov topology. Let $\tilde{S}_{4^{-n}}^{1}=\left\{(x, y) \in S^{1} \times S^{1} \mid \operatorname{dist}(x, y) \geqslant 4^{-n}\right\}$. Since for each $\left(x_{1}, y_{1}, x_{2}, y_{2}, \ldots\right) \in \tilde{\Sigma}$ and each $i$ we have

$$
\operatorname{dist}\left(x_{i+1}, y_{i+1}\right) \geqslant \frac{1}{3} \operatorname{dist}\left(x_{i}, y_{i}\right),
$$

there is $n$ such that $\operatorname{dist}\left(x_{n}, y_{n}\right)>4^{-n}$. Therefore it suffices to construct a sequence of equivariant maps $r_{n}: \tilde{S}_{4^{-n}}^{1} \rightarrow S^{1}$ such that $r_{n} \circ \tilde{p}=r_{n+1}$ over $\tilde{p}^{-1}\left(\tilde{S}_{4^{-n}}^{1}\right)$. Then we can define an equivariant map $r: \tilde{\Sigma} \rightarrow S^{1}$ by the formula $r\left(x_{1}, y_{1}, x_{2}, y_{2}, \ldots\right)=$ $r_{n}\left(x_{n}, y_{n}\right)$ for sufficiently great $n$. Since $\tilde{p} \tilde{S}_{4^{-n}}^{1} \supset \tilde{S}_{4^{-n+1}}^{1}$ and $r_{n} \circ \tilde{p}=r_{n+1}$, then $r$ is well-defined. For each $z \in \tilde{\Sigma}$ and an open neighborhood $U \subset S^{1}$ of $r(z)$, take $n$ such that $\operatorname{dist}\left(a_{n}, b_{n}\right)>4^{-n}$. Then $\left\{\left(x_{n}, y_{n}\right) \in \tilde{S}^{1} \mid \operatorname{dist}\left(x_{n}, y_{n}\right)>4^{-n}\right\}$ is an open subset of $\tilde{S}^{1}$, on which $r_{n}$ is defined. Hence $\left\{\left(x_{1}, y_{1}, x_{2}, y_{2}, \ldots\right) \in \tilde{\Sigma} \mid \operatorname{dist}\left(x_{n}, y_{n}\right)>\right.$ $\left.4^{-n}\right\}$ is an open neighborhood of $z$ in $\tilde{\Sigma}$, going to $U$ under $r$. Therefore $r$ is continuous.

We shall construct such maps $r_{n}$ successively. Let $r_{1}: \tilde{S}_{1 / 4}^{1} \rightarrow S^{1}$ be an arbitrary equivariant map. Suppose that $r_{n-1}$ is already constructed. For $M \subset S^{1}$ denote $A(M)=\left\{(x, y) \in \tilde{S}^{1} \mid \arg \frac{x}{y} \in M\right\}$. If $M$ is an interval with ends $a$, $b$, then $A(M)$ is the annulus with boundary circles $A(a), A(b)$. By the condition $r_{n}=r_{n-1} \circ \tilde{p}$ the map $r_{n}$ is already defined on the union of the three annuli (white on Figure 5)

$$
\begin{gathered}
A\left[\frac{1}{3 \cdot 4^{n-1}} ; \frac{2 \pi}{3}-\frac{1}{3 \cdot 4^{n-1}}\right] \cup A\left[\frac{2 \pi}{3}+\frac{1}{3 \cdot 4^{n-1}} ; \frac{4 \pi}{3}-\frac{1}{3 \cdot 4^{n-1}}\right] \\
\cup A\left[\frac{4 \pi}{3}+\frac{1}{3 \cdot 4^{n-1}} ; 2 \pi-\frac{1}{3 \cdot 4^{n-1}}\right] .
\end{gathered}
$$

Since $\left.r_{n}\right|_{A\left(\frac{1}{4^{n-1}}\right)}$ and $\left.r_{n}\right|_{A\left(2 \pi-\frac{1}{4^{n-1}}\right)}$ are homotopic, then

$$
\left.r_{n+1}\right|_{A\left(\frac{2 \pi}{3}-\frac{1}{3 \cdot 4^{n-1}}\right)} \text { and }\left.r_{n+1}\right|_{A\left(\frac{2 \pi}{3}+\frac{1}{3 \cdot 4^{n-1}}\right)}
$$

are homotopic. Therefore $r_{n}$ is extendable over $A\left[\frac{2 \pi}{3}-\frac{1}{3 \cdot 4^{n-1}}, \frac{2 \pi}{3}+\frac{1}{3 \cdot 4^{n-1}}\right]$. Hence $r_{n}$ is equivariantly extendable over $\tilde{S}_{4^{-n}}^{1}$. We take as $r_{n}: \tilde{S}_{4^{-n}}^{1} \rightarrow S^{1}$ any such extension. 


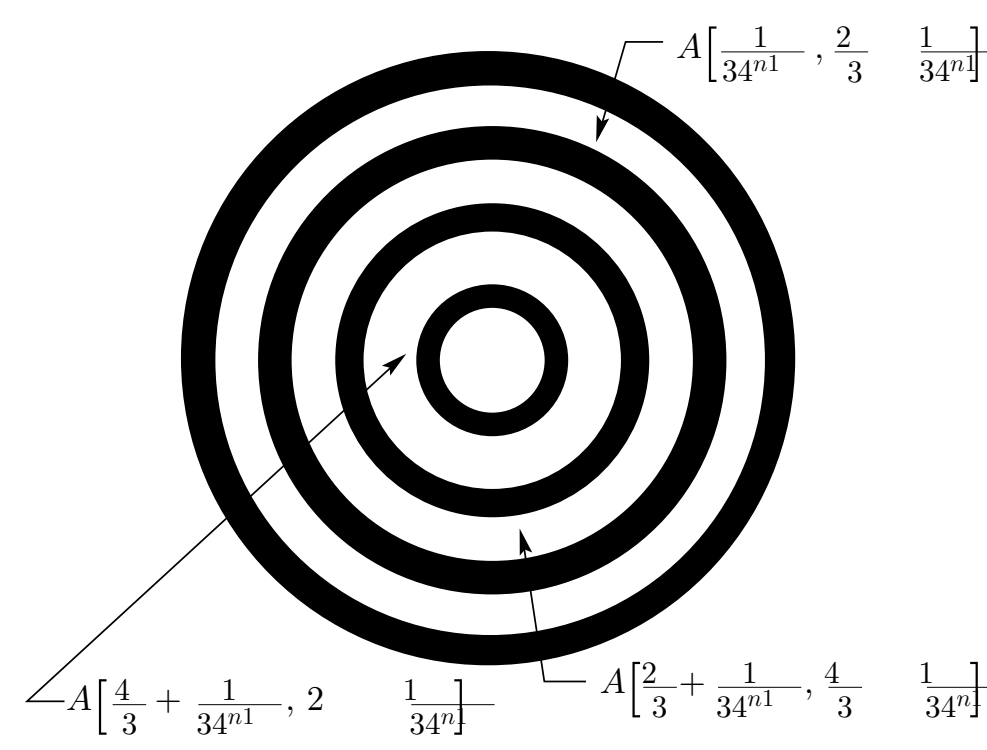

FiguRE 5

\section{ACKNOWLEDGMENTS}

I would like to thank V. S. Krushkal and E. V. Schepin for useful discussions. These results were first presented at The Alexandrov Session in Moscow, May 1995, and at The Borsuk-Kuratowski Session in Warsaw, May 1996.

\section{REFERENCES}

[Al 30] J. W. Alexander, The combinatorial theory of complexes, Ann. of Math. 31 (1930), 292-320.

[Cl 34] S. Claytor, Topological immersions of peanian continua in a spherical surface, Ann. of Math. (2) 35 (1934), 809-835.

[Cl 37] S. Claytor, Peanian continua not embeddable in a spherical surface, Ann. of Math. 38 (1937), 631-646.

[Co 69] M. M. Cohen, A general theory of relative regular neighbourhoods, TAMS 136 (1969), 189-230. MR 40:2052

[FKT 94] M. H. Freedman, V. S. Krushkal, P. Teichner, Van Kampen's embedding obstruction is incomplete for 2-complexes in $\mathbb{R}^{4}$, Math. Res. Letters 1 (1994), 167-176. MR 95c:57005

[Hae 63] A. Haefliger, Plongements differentiables dans le domain stable, Comment. Math. Helv. 37 (1962-63), 155-176. MR 28:625

[HJ 64] R. Halin and H. A. Jung, Karakterisierung der komplexe der Ebene und der 2-Sphäre, Arch. Math. 15 (1964), 466-469. MR 30:579

[Har 69] L.S.Harris, Intersections and embeddings of polyhedra, Topology 8 (1969), 1-26. MR 38:6602

[Hu 88] L. S. Husch, $\varepsilon$-maps and embeddings, Genegal Topological Relations to Modern Analysis and Algebra, VI, Heldermann, Berlin, 1988, pp. 273-280. MR 89g:54033

[Ku 30] K. Kuratowski, Sur le problemes des courbes gauche en topologie, Fund. Math. 15 (1930), 271-283.

[Li 65] R. Lickorish, The piecewise linear unknotting of cones, Topology 4 (1965), 67-91. MR 34:3585

[Ma 97] Yu. Makarychev, A short proof of Kuratowski's graph planarity criterion, J.of Graph Theory 25 (1997), 129-131. CMP 97:12

[MS 66] S. Mardešić and J. Segal, A note on polyhedra embeddable in the plane, Duke Math. J. 33 (1966), 633-638. MR 33:7988 
[MS 67] S. Mardesic, J. Segal, ع-mappings and generalized manifolds, Michigan Math. J. 14 (1967), 171-182. MR 35:2288

[PWZ 61] R. Penrose, J. H. C. Whitehead, E. C. Zeeman, Imbedding of manifolds in euclidean space, Ann. of Math. 73 (1961), 613-623. MR 23:A2218

[RS 72] C. P. Rourke, B. J. Sanderson, Introduction to Piecewise-Linear Topology, Ergebn. der Math. 69, Springer-Verlag, Berlin, 1972. MR 50:3236

[RS 96] D.Repovs, A.B.Skopenkov, Embeddability and isotopy of polyhedra in euclidean spaces, Proceedings of the Steklov Institute of Mathematics 212 (1996), 163-178.

[RS 97] D.Repovs, A.B.Skopenkov, A deleted product criterion for approximability of maps by embeddings, Topology Appl., to appear.

[Sa 91] K. S. Sarkaria, A one-dimensional Whitney trick and Kuratowski's graph planarity criterion, Israel J. Math. 73 (1991), 79-89. MR 92g:57034

[SS 92] J.Segal, S.Spiez, Quasi-embeddings and embeddings of polyhedra in $R^{m}$, Topol.Appl. 45 (1992), 275-282. MR 94d:57050

[SSS 97] J.Segal, A.Skopenkov, S.Spiez, Embedding of polyhedra in $R^{m}$ and the deleted product obstruction, Topol.Appl. 85 (1997).

[Sk 95] A. B. Skopenkov, On the deleted product obstruction for embeddability of polyhedra in $\mathbb{R}^{m}$, Abstracts AMS (1995), 95T-57-94.

[Sk 97] A. B. Skopenkov, On the deleted product criterion for embeddability of manifolds in $\mathbb{R}^{m}$, Comment. Math. Helv. 72 (1997), 543-555.

[Th 81] C.Thomassen, Kuratowski's theorem, J.of Graph Theory 5 (1981), 225-241. MR 83d:05039

[We 67] C. Weber, Plongements de polyedres dans le domaine metastable, Comment. Math. Helv. 42 (1967), 1-27. MR 38:6606

[We 74] C. Weber, $\epsilon$-applications dans une variété, Comment. Math. Helv. 49 (1974), 125-135. MR 49:3955

[Wu 65] W.T.Wu, A Theory of Embeddings, Isotopy and Immersions of Polyhedra in Euclidean Space, Science Press, Peking (1965).

[Ze 63] E. C. Zeeman, Unknotting combinatorial balls, Ann. Math. 78 (1963), 501-526. MR 28:3432

[Ze 66] E. C. Zeeman, Notes on combinatorial topology (mimeographed), IHES, Paris, 1963, revised 1966 .

Chair of Differential Geometry, Department of Mechanics and Mathematics, Moscow State University, Moscow,119899, Russia

E-mail address: skopenko@nw.math.msu.su 